\title{
Subcritical Hopf Equilibrium Points in the Boundary of the Stability Region ${ }^{\dagger}$
}

\author{
J.R.R. GOUVEIA Jr ${ }^{1 *}$, F.M. AMARAL ${ }^{1}$ and L.F.C. ALBERTO ${ }^{2}$
}

Received on December 20, 2015 / Accepted on May 11, 2016

\begin{abstract}
A complete characterization of the boundary of the stability region of a class of nonlinear autonomous dynamical systems is developed admitting the existence of Subcritical Hopf nonhyperbolic equilibrium points on the boundary of the stability region. The characterization of the stability region developed in this paper is an extension of the characterization already developed in the literature, which considers only hyperbolic equilibrium point. Under the transversality condition, it is shown the boundary of the stability region is comprised of the stable manifolds of all equilibrium points on the boundary of the stability region, including the stable manifolds of the subcritical Hopf equilibrium points of type $k$, with $0 \leq k \leq n-2$, which belong to the boundary of the stability region.
\end{abstract}

Keywords: dynamical systems, nonlinear systems, stability region, boundary of the stability region, subcritical Hopf equilibrium point.

\section{INTRODUCTION}

Dynamic and topological characterizations of the boundary of the stability regions of autonomous nonlinear dynamic systems were developed, for example in $[3,5]$. Those characterizations were derived under some assumptions over the vector field, including hyperbolicity of equilibrium points on the boundary of the stability region and transversality conditions.

Although the hyperbolicity of equilibrium points of a dynamical system is a generic property, that is, it is satisfied for almost all dynamic systems, violation of the hyperbolicity condition of equilibrium points on the boundary of the stability region commonly occurs when the system is subject to variations of parameters. With this variation of parameters, the occurrence of local bifurcations of equilibrium points on the boundary of the stability region is common.

\footnotetext{
$\dagger$ Paper presented at the Third Congress of Applied and Computational Mathematics of the Southeast Region. *Corresponding author: Josaphat Ricardo Ribeiro Gouveia Jr.

${ }^{1}$ Departamento de Ensino, Instituto Federal de Educação, Ciência e Tecnologia da Bahia, 45822-000 Eunápolis, BA, Brasil. E-mails: josaphat@ifba.edu.br; fabiolo@ifba.edu.br

2 Departamento de Engenharia Elétrica e de Computação, EESC - USP, Av. Trabalhador Sancarlense, 400, $13566-590$ São Carlos, SP, Brasil. E-mail: 1fcalberto@usp.br
} 
In this paper, we are interested in studying the characterization of the stability region and its boundary when the hyperbolicity condition on the boundary is violated due to the presence of nonhyperbolic equilibrium points. Some advances in this direction have already been obtained and reported in the literature. A complete characterization of the boundary of the stability region in the presence of saddle-node equilibrium points was developed in [1]. A complete characterization was also developed considering type- $k$ supercritical Hopf equilibrium points, with $k \geq 1$, on the boundary [9].

In this paper, a complete characterization of the stability boundary is developed admitting the existence of type- $k$ subcritical Hopf nonhyperbolic equilibrium points, with $k \geq 1$, on the boundary. More precisely, if $x^{s}$ is an asymptotically stable equilibrium point and $A\left(x^{s}\right)$ is its stability region, it is proven in this paper, under mild assumptions, that:

$$
\partial A\left(x^{s}\right)=\bigcup_{i} W^{s}\left(x_{i}\right) \bigcup_{j} W^{s}\left(p_{j}\right)
$$

that is, the stability boundary $\partial A\left(x^{s}\right)$ is comprised of the union of all stable manifolds of the hyperbolic equilibrium points lying on the stability boundary union with the stable manifolds of the subcritical Hopf equilibrium points on the stability boundary. This characterization will help us to understand the mechanisms of Hopf bifurcations on the stability boundary and their implication on the stability region and its changes with respect to parameter variations.

This article is organized as follows. In Section 2, a review of the characterization of the boundary of the stability region of nonlinear autonomous dynamic systems is presented. In Section 3, the subcritical Hopf equilibrium points are studied and the local dynamics on the neighborhood of these points is reviewed. The main contribution of this paper is presented in Section 4.

\section{PRELIMINARIES}

In this section, we review some classic concepts related to the theory of dynamical systems, which are essential for the further developments of this work. More details on the contents explored in this section can be found at $[18,15]$.

Consider the nonlinear autonomous dynamic system:

$$
\dot{x}=f(x)
$$

where $x \in \mathbb{R}^{n}$ and $f: \mathbb{R}^{n} \rightarrow \mathbb{R}^{n}$ is a smooth vector field. We use the term smooth to refer to a field whose differentiability class is large enough, namely a vector field of class $C^{r}$ with $r \geq 1$. The solution of (2.1) starting at $x$ at time $t=0$ is denoted by $\varphi(t, x)$.

Suppose that $x^{s}$ is an asymptotically stable equilibrium point of system (2.1). The stability region (or region of attraction) of $x^{s}$ is the set $A\left(x^{s}\right)=\left\{x \in \mathbb{R}^{n} \mid \varphi(t, x) \rightarrow x^{s}\right.$ as $\left.t \rightarrow+\infty\right\}$, of all initial conditions $x \in \mathbb{R}^{n}$ whose trajectories converge to $x^{s}$ when $t$ tends to infinity. The stability region $A\left(x^{s}\right)$ is an open and invariant set. Its closure $\overline{A\left(x^{s}\right)}$ is invariant and the boundary of the stability region $\partial A\left(x^{s}\right)$ is a closed and invariant set. 
With the motivation of better understanding the boundary of the stability region and getting better estimates of the stability region, characterizations of the boundary of the stability region were developed.

The first characterization of the boundary of the stability region of an asymptotically stable equilibrium point $x^{s}$ of system (2.1) was developed in [14]. A generalization of the characterization proposed in [14] was developed in [4], under the following assumptions:

(A1) All the equilibrium points on $\partial A\left(x^{s}\right)$ are hyperbolic;

(A2) The stable and unstable manifolds of equilibrium points on $\partial A\left(x^{s}\right)$ satisfy the transversality condition;

(A3) Trajectories on $\partial A\left(x^{s}\right)$ approach one of the equilibrium points as $t \rightarrow \infty$.

The boundary of the stability region of an asymptotically stable equilibrium point $x^{s}$ of system (2.1), satisfying assumptions (A1), (A2) and (A3), is the union of all stable manifolds of the equilibrium points on the boundary, in other words $\partial A\left(x^{s}\right)=\bigcup_{i} W^{s}\left(x_{i}\right)$, where $x_{i}, i=1,2, \ldots$ are the hyperbolic equilibrium points on the stability boundary $\partial A\left(x^{s}\right)$.

Assumption (A3) is not a generic property of dynamical systems and needs to be checked [3]. Sufficient conditions for the satisfaction of assumption (A3) were given in [3]. The existence of an energy function is a sufficient condition to guarantee the fulfilment of assumption (A3), and, consequently, a fairly large class of dynamical systems satisfy this condition, see [3].

Although assumption (A1) is generic, see [11], studying the characterization of the stability boundary in the presence of non-hyperbolic equilibrium points is important to understand how the stability region changes as a consequence of parameter variations. These changes were already investigated in the occurrence of type-zero saddle-node bifurcations on the stability boundary [1], [2] and in the occurrence of type- $k$ supercritical Hopf equilibrium points, with $1 \leq k \leq n-2$, [8,9].

In this paper, we also study the characterization of the boundary of the stability region when assumption (A1) is violated. More specifically, we study the characterization of the stability boundary when a subcritical Hopf non-hyperbolic equilibrium point is found on the stability boundary.

\section{SUBCRITICAL HOPF EQUILIBRIUM POINT}

In this section, a particular type of non-hyperbolic equilibrium point, namely the subcritical Hopf equilibrium point, is studied. Particularly, the dynamic behavior in a neighborhood of this equilibrium is explored in details and also the asymptotic behavior of solutions in the invariant local manifolds is discussed.

Consider the nonlinear dynamical system (1). An equilibrium point $x^{\star}$ of (2.1) is said to be hyperbolic if all the eigenvalues of the Jacobian matrix $D_{x} f\left(x^{\star}\right)$ do not have null real part. 
Furthermore, a hyperbolic equilibrium point $x^{\star}$ is of type- $k$ if the Jacobian matrix possesses $k$ eigenvalues with positive real part and $n-k$ eigenvalues with negative real part. A non-hyperbolic equilibrium point $p \in \mathbb{R}^{n}$ of (1) is called a Hopf equilibrium point if the following conditions are satisfied:

(i) $D_{x} f(p)$ has a simple pair of purely imaginary eigenvalues, $\pm i \omega$, and no other eigenvalue with null real part;

(ii) $l_{1} \neq 0$, where $l_{1}$ is the first Lyapunov coefficient, see $[8,9]$.

Lyapunov coefficients indicate the level of degeneration of the vector field. If the first Lyapunov coefficient is non-zero, then the vector field has a degeneration of cubic order showing that cubic terms are those that determine the type of dynamic behavior location in the neighborhood of the non-hyperbolic equilibrium point in the cental manifold, see [18] for more details.

Hopf equilibrium points can be classified according to the sign of the first Lyapunov coefficient. A Hopf equilibrium point $p \in \mathbb{R}^{n}$ of (2.1) is called a supercritical Hopf equilibrium point if the first Lyapunov coefficient $l_{1}<0$ and is called a subcritical Hopf equilibrium point if the first Lyapunov coefficient $l_{1}>0$.

Hopf equilibrium points can be also classified in types according to the number of eigenvalues of $D_{x} f(p)$ with positive real part. A Hopf equilibrium point $p$ of (2.1) is called a type- $k$ Hopf equilibrium point if $D_{x} f(p)$ has $k(k \leq n-2)$ eigenvalues with positive real part and $n-k-2$ with negative real part.

In this paper, we are primarily concerned with subcritical Hopf equilibium points. If $p$ is a subcritical Hopf equilibrium point, then the following properties are satisfied, see $[18,10]$ :

(1) $p$ is a type-0 subcritical Hopf equilibrium point of (2.1):

(i) The $(n-2)$-dimensional local stable manifold $W_{l o c}^{s}(p)$ of $p$ exists, is unique, and if $q \in W_{l o c}^{s}(p)$ then $\varphi(t, q) \longrightarrow p$ as $t \longrightarrow+\infty$.

(ii) The bidimensional local center manifold $W_{l o c}^{c}(p)$ of $p$ exists, is unique, and if $q \in$ $W_{l o c}^{c}(p)$ then $\varphi(t, q) \longrightarrow p$ as $t \longrightarrow-\infty$.

(2) $p$ is a type- $k$ subcritical Hopf equilibrium point of (2.1), with $1 \leq k \leq n-3$ :

(i) The $k$-dimensional local unstable manifold $W_{l o c}^{u}(p)$ of $p$ exists, is unique, and if $q \in$ $W_{l o c}^{u}(p)$ then $\varphi(t, q) \longrightarrow p$ as $t \longrightarrow-\infty$.

(ii) The $(n-k-2)$-dimensional local stable manifold $W_{l o c}^{s}(p)$ of $p$ exists, is unique, and if $q \in W_{l o c}^{s}(p)$ then $\varphi(t, q) \longrightarrow p$ as $t \longrightarrow+\infty$.

(iii) The $(k+2)$-dimensional local unstable center manifold $W_{l o c}^{c u}(p)$ of $p$ exists, is unique, and if $q \in W_{l o c}^{c s}(p)$ then $\varphi(t, q) \longrightarrow p$ as $t \longrightarrow-\infty$.

(3) $p$ is a type- $(n-2)$ subcritical Hopf equilibrium point of (2.1): 
(i) The $(n-2)$-dimensional local unstable manifold $W_{l o c}^{u}(p)$ of $p$ exists, is unique, and if $q \in W_{l o c}^{u}(p)$ then $\varphi(t, q) \longrightarrow p$ as $t \longrightarrow-\infty$.

(ii) The bidimensional local center manifold $W_{l o c}^{c}(p)$ of $p$ exists, is not unique, and if $q \in W_{l o c}^{c}(p)$ then $\varphi(t, q) \longrightarrow p$ as $t \longrightarrow-\infty$.

Figure 1.1(a) illustrates the invariant manifolds for a type-1 subcritical Hopf equilibrium point in $\mathbb{R}^{3}$ and Figure 1.1(b) illustrates these invariant manifolds for a type-0 subcritical Hopf equilibrium point in $\mathbb{R}^{3}$.

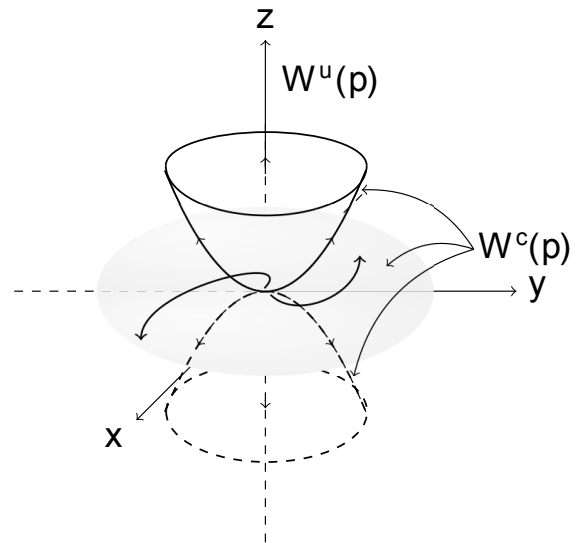

(a) Manifolds $W^{c}(p)$ and $W^{s}(p)$ for a type-1 subcritical Hopf equilibrium point $p$ of system (2.1) in $\mathbb{R}^{3} \cdot W^{c}(p)$ is not unique. Three choices of $W^{c}(p)$ are displayed in this figure.

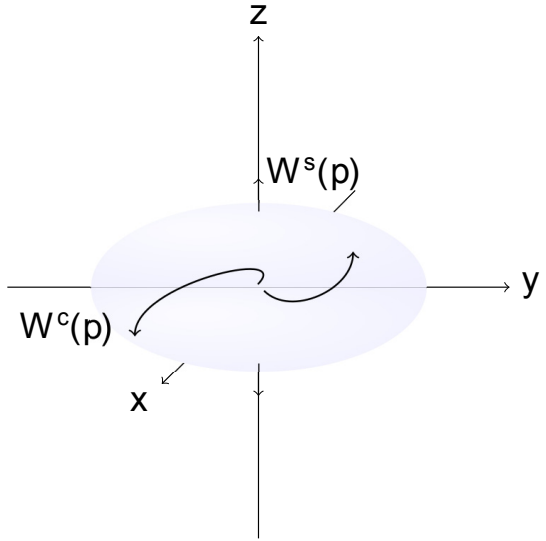

(b) Manifolds $W^{c}(p)$ and $W^{u}(p)$ for a type- 0 subcritical Hopf equilibrium point $p$ of system (2.1) in $\mathbb{R}^{3}$. In this case, $W^{c}(p)$ is unique.

Figure 1

The stable and unstable manifolds of a hyperbolic equilibrium point are defined by extending the local manifolds through the flow, see [16]. Often, this technique to define the global manifolds cannot be applied to general non-hyperbolic equilibrium points. Even though, in the particular case of subcritical Hopf equilibrium points, one can also define the global manifolds $W^{s}(p)$, $W^{u}(p), W^{c}(p)$ and $W^{c u}(p)$ by extending the local manifolds $W_{l o c}^{s}(p), W_{l o c}^{u}(p), W_{l o c}^{c}(p)$ and $W_{l o c}^{c u}(p)$ through the flow.

\section{SUBCRITICAL HOPF EQUILIBRIUM POINT ON THE STABILITY BOUNDARY}

In this section, results of characterization of equilibrium points on the boundary of the stability region are presented. The characterization of the boundary of stability region in the presence of a subcritical Hopf equilibrium point will be developed in two steps. First we study a local characterization of the stability boundary by studying and characterizing the equilibrium points that belong to the stability boundary, then a global characterization on the boundary is developed. 
The next theorems provide necessary and sufficient conditions to guarantee that a subcritical Hopf equilibrium point lies on the boundary of the stability region in terms of the properties of its stable, center-unstable and center manifolds.

Theorem 4.1. (Subcritical Hopf equilibrium point on $\partial A\left(x^{s}\right)$ ) Let $p$ be a subcritical Hopf equilibrium point of (2.1). Suppose also, the existence of an asymptotically stable equilibrium point $x^{s}$ and let $A\left(x^{s}\right)$ be its stability region. Then the following holds:

(i) if $p$ is a type-0 subcritical Hopf equilibrium point of (2.1), then:

$$
\begin{aligned}
& p \in \partial A\left(x^{s}\right) \Longleftrightarrow\left(W_{l o c}^{c}(p) \backslash\{p\}\right) \cap \overline{A\left(x^{s}\right)} \neq \varnothing \\
& p \in \partial A\left(x^{s}\right) \Longleftrightarrow W_{l o c}^{s}(p) \cap \partial A\left(x^{s}\right) \neq \emptyset
\end{aligned}
$$

(ii) if $p$ is a type- $k$ subcritical Hopf equilibrium point of (2.1), with $1 \leq k \leq n-3$, then:

$$
\begin{aligned}
& p \in \partial A\left(x^{s}\right) \Longleftrightarrow\left(W_{l o c}^{c u}(p) \backslash\{p\}\right) \cap \overline{A\left(x^{s}\right)} \neq \varnothing \\
& p \in \partial A\left(x^{s}\right) \Longleftrightarrow W_{l o c}^{s}(p) \cap \partial A\left(x^{s}\right) \neq \emptyset
\end{aligned}
$$

(iii) if $p$ is a type- $(n-2)$ subcritical Hopf equilibrium point of (2.1), then:

$$
p \in \partial A\left(x^{s}\right) \Longleftrightarrow\left(W_{l o c}^{c u}(p) \backslash\{p\}\right) \cap \overline{A\left(x^{s}\right)} \neq \emptyset
$$

Proof. (i) $(\Longleftarrow)$ Suppose that $\left(W_{l o c}^{c}(p) \backslash\{p\}\right) \cap \overline{A\left(x^{s}\right)} \neq \emptyset$. Then there exists $q \in\left(W_{l o c}^{c}(p) \backslash\right.$ $\{p\}) \cap \overline{A\left(x^{s}\right)}$. Observe that $\varphi(t, q) \longrightarrow p$ as $t \longrightarrow-\infty$. On the other hand, set $\overline{A\left(x^{s}\right)}$ is invariant thus, $\varphi(t, q) \in \overline{A\left(x^{s}\right)}$ for all $t \leq 0$. Consequently $p \in \overline{A\left(x^{s}\right)}$, since $\overline{A\left(x^{s}\right)}$ is closed. Since $p \notin A\left(x^{s}\right)$, we have that $p \in \mathbb{R}^{n} \backslash A\left(x^{s}\right)$. Therefore, $p \in \partial A\left(x^{s}\right)$. Now suppose that $W_{l o c}^{s}(p) \cap \partial A\left(x^{s}\right) \neq \emptyset$. Therefore, there exists $q \in W_{l o c}^{s}(p) \cap \partial A\left(x^{s}\right)$. Note that $\varphi(t, q) \longrightarrow p$ as $t \longrightarrow+\infty$. Since set $\partial A\left(x^{s}\right)$ is invariant and $q \in \partial A\left(x^{s}\right)$, thus $\varphi(t, q) \in \partial A\left(x^{s}\right)$ for all $t \geq 0$. Since $\partial A\left(x^{s}\right)$ is closed, thus $p \in \partial A\left(x^{s}\right)$.

$(\Longrightarrow)$ Suppose that $p \in \partial A\left(x^{s}\right)$. Let $N^{c}$ be a fundamental domain of $W^{c}(p)$, that is, $\bigcup_{t \in \mathbb{R}} \varphi\left(t, N^{c}\right)=W^{c}(p) \backslash\{p\}$. Let $N_{\varepsilon}^{c}$ be a fundamental neighborhood of radius $\varepsilon$ of $N^{c}$, namely $N_{\varepsilon}^{c}=\left\{x \in \mathbb{R}^{n}: d\left(x, N^{c}\right)<\varepsilon\right\}$. As a consequence of $\lambda$-lemma, see [6], there exists a neighborhood $U$ of $p$ such that $\bigcup_{t \leq 0} \varphi\left(t, N_{\varepsilon}^{c}\right) \supset U \backslash W_{l o c}^{s}(p)$. Since $p \in \partial A\left(x^{s}\right)$, then $U \cap A\left(x^{s}\right) \neq \emptyset$. On the other hand, $W_{l o c}^{s}(p) \cap A\left(x^{s}\right)=\emptyset$. Thus, $\left\{U \backslash W_{l o c}^{s}(p)\right\} \cap A\left(x^{s}\right) \neq \emptyset$. Consequently, there is a point $z \in N_{\varepsilon}^{c}$ and a time $\bar{t}$ such that $\varphi(\bar{t}, z) \in A\left(x^{s}\right)$. Since $A\left(x^{s}\right)$ is invariant, then $z \in A\left(x^{s}\right)$. As $\varepsilon$ can be chosen arbitrarily small, we can find a sequence of points $\left\{z_{i}\right\}$ with $z_{i} \in A\left(x^{s}\right)$ for all $i=1,2, \ldots$ such that $d\left(z_{i}, N^{u}\right) \rightarrow 0$ when $i \rightarrow+\infty$. By construction, the sequence $\left\{z_{i}\right\}$ is bounded and therefore has a convergent subsequence. Let $\left\{z_{i_{k}}\right\}$ be a convergent subsequence, that is $z_{i_{k}} \rightarrow \bar{z}$ when $i_{k} \rightarrow+\infty$. Observe that $d\left(z_{i_{k}}, N^{c}\right) \rightarrow d\left(\bar{z}, N^{c}\right)$ when $i_{k} \rightarrow+\infty$ and, therefore, $\bar{z} \in \overline{N^{c}} \subset W_{l o c}^{c}(q) \backslash\{q\}$. Thus,

$$
\bar{z} \in\left(W_{l o c}^{c}(p) \backslash\{p\}\right) \cap \overline{A\left(x^{s}\right)} .
$$

The proof that $W_{l o c}^{s}(p) \cap \partial A\left(x^{s}\right) \neq \emptyset$ if $p \in \partial A\left(x^{s}\right)$ is very similar to the previous one and therefore will be omitted.

The proofs of (ii) and (iii) are similar to the proof of (i) and will also be omitted. 
Theorem 4.1, besides being relevant for the development of a complete characterization of the stability boundary in the presence of subcritical Hopf equilibrium points on the stability boundary, provides a way of checking if a supercritical Hopf equilibrium point lies on the stability boundary by checking if its center and center-unstable manifold intersects the stability region. A numerical algorithm for checking this condition was suggested in [3].

As a consequence of Theorem 4.1, we know that $W_{l o c}^{c u}(p) \cap A\left(x^{s}\right) \neq \emptyset$ is a sufficient condition to guarantee that the subcritical Hopf equilibrium point $p$ lies on the stability boundary. It will be relevant, for the sake of developing a characterization of the stability boundary, verifying when this condition is also necessary.

Items (i) and (ii) of Theorem 4.1 can be improved if we impose some conditions to the vector field. Let $x^{s}$ be an asymptotically stable equilibrium point and consider the following assumptions:

(A1") All the equilibrium points on $\partial A\left(x^{s}\right)$ are hyperbolic or subcritical Hopf equilibrium points.

(A2") The stable, the unstable, the center-unstable and/or the center manifold of the equilibrium points on $\partial A\left(x^{s}\right)$ satisfy the transversality condition.

It is worth mentioning that condition (A1") is weaker than condition (A1), since it allows the presence of non-hyperbolic subcritical Hopf equilibrium points on the stability boundary. The next results provide necessary and sufficient conditions to guarantee that the hyperbolic equilibrium points and subcritical Hopf equilibrium points belong to the boundary of the stability region. Initially, we provide these conditions for type- 1 hyperbolic equilibrium points and typezero subcritical Hopf equilibrium points on the boundary of the stability region and then these conditions for equilibrium points of types higher than 1 follow by arguments of induction.

Theorem 4.2. Let $A\left(x^{s}\right)$ be the stability region of an asymptotically stable equilibrium point $x^{s}$ of (2.1). Let $x^{\star}$ be a hyperbolic equilibrium point and $p$ be a subcritical Hopf equilibrium point of (2.1). If assumptions (A1"), (A2") and (A3) are held, then:

1. if $x^{\star}$ is a type-1 or a type-2 hyperbolic equilibrium point, then

(i) $x^{\star} \in \partial A\left(x^{s}\right) \Longleftrightarrow W^{u}\left(x^{\star}\right) \cap A\left(x^{s}\right) \neq \varnothing$

(ii) $x^{\star} \in \partial A\left(x^{s}\right) \Longleftrightarrow W^{s}\left(x^{\star}\right) \subset \partial A\left(x^{s}\right)$

2. if $p$ is a type-0 subcritical Hopf equilibrium point, then

(i) $p \in \partial A\left(x^{s}\right) \Longleftrightarrow W^{c}(p) \cap A\left(x^{s}\right) \neq \emptyset$

(ii) $p \in \partial A\left(x^{s}\right) \Longleftrightarrow W^{s}(p) \subset \partial A\left(x^{s}\right)$

3. if $p$ is a type-1 subcritical Hopf equilibrium point

(i) $p \in \partial A\left(x^{s}\right) \Longleftrightarrow W^{c u}(p) \cap A\left(x^{s}\right) \neq \emptyset$

(ii) $p \in \partial A\left(x^{s}\right) \Longleftrightarrow W^{s}(p) \subset \partial A\left(x^{s}\right)$ 
Proof. 1(i) $(\Longleftarrow)$ Suppose that $W^{u}\left(x^{\star}\right) \cap A\left(x^{s}\right) \neq \emptyset$. Since $A\left(x^{s}\right) \subset \overline{A\left(x^{s}\right)}$, then $\left(W_{l o c}^{u}\left(x^{\star}\right) \backslash\right.$ $\left.\left\{x^{\star}\right\}\right) \cap \overline{A\left(x^{s}\right)} \neq \emptyset$. Therefore, by Theorem 3.7 of [3], we have that $x^{\star} \in \partial A\left(x^{s}\right)$.

$(\Longrightarrow)$ Suppose that $x^{\star} \in \partial A\left(x^{s}\right)$. By Theorem 3.7 of [3], we can conclude that $\left(W_{l o c}^{u}\left(x^{\star}\right) \backslash\right.$ $\left.\left\{x^{\star}\right\}\right) \cap \overline{A\left(x^{s}\right)} \neq \emptyset$. Consequently, $\left(W^{u}\left(x^{\star}\right) \backslash\left\{x^{\star}\right\}\right) \cap \overline{A\left(x^{s}\right)} \neq \emptyset$, because $W_{l o c}^{u}\left(x^{\star}\right) \subset W^{u}\left(x^{\star}\right)$. Let us show, under assumptions (A1"), (A2") and (A3) that $\left(W^{u}\left(x^{\star}\right) \backslash\left\{x^{\star}\right\}\right) \cap \overline{A\left(x^{s}\right)} \neq \varnothing$ implies $W^{u}\left(x^{\star}\right) \cap A\left(x^{s}\right) \neq \emptyset$. Let $q \in\left(W^{u}\left(x^{\star}\right) \backslash\left\{x^{\star}\right\}\right) \cap \overline{A\left(x^{s}\right)}$. If $q \in A\left(x^{s}\right)$, then there is nothing to be proved. Suppose that $q \in \partial A\left(x^{S}\right)$. From condition (A3), there is an equilibrium point $\widehat{p} \in$ $\partial A\left(x^{s}\right)$ such that $\varphi(t, q) \rightarrow \widehat{p}$ as $t \rightarrow+\infty$. By supposition (A1"), $\widehat{p}$ is a hyperbolic equilibrium point or a subcritical Hopf equilibrium point. By the dimension of the unstable manifold of the equilibrium point, see [7], we conclude that $\operatorname{dim} W^{c u}(\widehat{p})<\operatorname{dim} W^{u}\left(x^{\star}\right)$ or $\operatorname{dim} W^{c}(\widehat{p})<$ $\operatorname{dim} W^{u}\left(x^{\star}\right)$ if $\widehat{p}$ is a subcritical Hopf equilibrium point or $\operatorname{dim} W^{u}(\widehat{p})<\operatorname{dim} W^{u}\left(x^{\star}\right)$ if $\widehat{p}$ is a hyperbolic equilibrium point.

Let $x^{\star}$ be a type-1 hyperbolic equilibrium point. Consequently, $\operatorname{dim} W^{u}(\widehat{p})<1$. Hence $\operatorname{dim} W^{u}(\widehat{p})=0$ and consequently $\widehat{p}$ is a type-zero hyperbolic equilibrium point. This leads us to a contradiction, because these type-zero equilibrium points cannot belong to $\partial A\left(x^{s}\right)$. Hence, $q \in A\left(x^{s}\right)$ and therefore, $W^{u}\left(x^{\star}\right) \cap A\left(x^{s}\right) \neq \emptyset$.

Let $x^{\star}$ be a type-2 hyperbolic equilibrium point. If $\widehat{p}$ is a subcritical Hopf equilibrium point, then $\operatorname{dim} W^{c u}(\widehat{p})<2$ or $\operatorname{dim} W^{c}(\widehat{p})<2$, which is a contradiction since the central manifold of a subcritical Hopf equilibrium point has at least dimension 2. Let $\widehat{p}$ be a hyperbolic equilibrium point and, therefore, $\operatorname{dim} W^{u}(\widehat{p})<2$. It follows that $\operatorname{dim} W^{u}(\widehat{p})=1$, since hyperbolic equilibrium points of type-zero can not belong to the boundary of the stability region. Therefore, $W^{u}(\widehat{p}) \cap A\left(x^{s}\right) \neq \emptyset$. Let $y \in W^{u}(\widehat{p}) \cap A\left(x^{s}\right)$ and $B(y, \varepsilon)$ be an open ball of radius $\varepsilon>0$ centered at $y$. Since $A\left(x^{s}\right)$ is an open set, then $B(y, \varepsilon) \subset A\left(x^{s}\right)$ for $\varepsilon$ sufficiently small. Let $N^{c}$ be a neighborhood of $q$ at $W^{u}\left(x^{\star}\right)$. The neighborhood $N^{c}$ contains a transversal section $D$ of $W^{s}(\widehat{p})$ at the point $q$ with $\operatorname{dimension} \operatorname{dim} D=1$. By $\lambda$-lemma, see [6], there is a point $w \in D$ and a time $t_{w}>0$ such that $\varphi\left(t_{w}, w\right) \in N^{c}$. Since $A\left(x^{s}\right)$ is an invariant set, then $w \in A\left(x^{s}\right)$. Therefore, $w \in W^{u}\left(x^{\star}\right) \cap A\left(x^{s}\right)$ and, consequently, $W^{u}\left(x^{\star}\right) \cap A\left(x^{s}\right) \neq \emptyset$.

1(ii) $(\Longleftarrow)$ Suppose that $W^{s}\left(x^{\star}\right) \subset \partial A\left(x^{s}\right)$. Since $x^{\star} \in W^{s}\left(x^{\star}\right)$, then $x^{\star} \in \partial A\left(x^{s}\right)$.

$(\Longrightarrow)$ Suppose now that $x^{\star} \in \partial A\left(x^{s}\right)$. By item 1(i) of Theorem 4.2, we conclude that $W^{u}\left(x^{\star}\right) \cap$ $A\left(x^{s}\right) \neq \emptyset$. Let $y \in W^{u}\left(x^{\star}\right) \cap A\left(x^{s}\right)$. Since $y \in W^{u}\left(x^{\star}\right)$, then there is $T<0$ such that $\varphi(T, y) \in W_{l o c}^{u}\left(x^{\star}\right)$. Let $z=\varphi(T, y)$. As $y \in A\left(x^{s}\right)$ and $A\left(x^{s}\right)$ is an invariant set, then $z \in A\left(x^{s}\right)$. It follows that $z \in W_{l o c}^{u}\left(x^{\star}\right) \cap A\left(x^{s}\right)$. Let $B(z, \varepsilon)$ be an open ball of radius $\varepsilon>0$ centered at $z$ where $\varepsilon$ is an arbitrarily small number. Let $\widehat{q}$ be an arbitrary point of $W^{s}\left(x^{\star}\right)$. In particular, for some $\bar{T}>0$ we have $\tilde{q}=\varphi(\bar{T}, \widehat{q}) \in W_{l o c}^{s}\left(x^{\star}\right)$. Let $S$ be a disk at point $\widetilde{q}$ of $\operatorname{dim} S=1$ or $\operatorname{dim} S=2$ transverse to $W_{l o c}^{S}\left(x^{\star}\right)$, if $x^{\star}$ is a type-1 or a type-2 hyperbolic equilibrium point, respectively. By $\lambda$-lemma, see [6], there is a point $w \in S$ and a time $t_{w}>0$ such that $\varphi\left(t_{w}, w\right) \in B(z, \varepsilon)$. Since $A\left(x^{s}\right)$ is an invariant set, then $w \in A\left(x^{s}\right)$. As $\varepsilon$ and the disk $S$ can be chosen arbitrarily small, then there are points at $A\left(x^{s}\right)$ arbitrarily close to $\widetilde{q}$. Consequently, $\widetilde{q} \in \overline{A\left(x^{s}\right)}$. Since $W_{l o c}^{s}\left(x^{\star}\right) \cap A\left(x^{s}\right)=\emptyset$, then $\widetilde{q} \in \partial A\left(x^{s}\right)$. As $\partial A\left(x^{s}\right)$ is 
invariant, $\widehat{q}=\varphi(-\bar{T}, \widetilde{q}) \in \partial A\left(x^{s}\right)$. As the choice of $\widehat{q}$ at $W^{s}\left(x^{\star}\right)$ was arbitrary, then we can conclude that $W^{s}\left(x^{\star}\right) \subset \partial A\left(x^{s}\right)$.

2(i) $(\Longleftarrow)$ Suppose that $W^{c}(p) \cap A\left(x^{s}\right) \neq \emptyset$. Since $A\left(x^{s}\right) \subset \overline{A\left(x^{s}\right)}$, then $\left(W_{l o c}^{c}(p) \backslash\{p\}\right) \cap$ $\overline{A\left(x^{s}\right)} \neq \emptyset$. Therefore, by item (i) of Theorem 4.1, we have that $p \in \partial A\left(x^{s}\right)$.

$(\Longrightarrow)$ Suppose that $p \in \partial A\left(x^{s}\right)$. By Theorem 4.1, we can conclude that $\left(W_{l o c}^{c}(p) \backslash\{p\}\right) \cap$ $\overline{A\left(x^{s}\right)} \neq \varnothing$. Consequently, $\left(W^{c}(p) \backslash\{p\}\right) \cap \overline{A\left(x^{s}\right)} \neq \varnothing$, because $W_{l o c}^{c}(p) \subset W^{c}(p)$. Let us show, under assumptions (A1"), (A2") and (A3) that $\left(W^{c}(p) \backslash\{p\}\right) \cap \overline{A\left(x^{s}\right)} \neq \varnothing$ implies $W^{c}(p) \cap A\left(x^{s}\right) \neq \emptyset$. Let $q \in\left(W^{c}(p) \backslash\{p\}\right) \cap \overline{A\left(x^{s}\right)}$. If $q \in A\left(x^{s}\right)$, then there is nothing to be proved. Suppose that $q \in \partial A\left(x^{s}\right)$. From condition (A3), there is an equilibrium point $\widehat{p} \in \partial A\left(x^{s}\right)$ such that $\varphi(t, q) \rightarrow \widehat{p}$ when $t \rightarrow+\infty$. By the dimension of the unstable manifold of the equilibrium point, see [7], we conclude that $\widehat{p} \neq p$. Then $\widehat{p}$ is a hyperbolic equilibrium point or a subcritical Hopf equilibrium point and we conclude that $\operatorname{dim} W^{c u}(\widehat{p})<\operatorname{dim} W^{c u}(p)$ or $\operatorname{dim} W^{c}(\widehat{p})<\operatorname{dim} W^{c u}(p)$, if $\widehat{p}$ is a subcritical Hopf equilibrium point or $\operatorname{dim} W^{u}(\widehat{p})<$ $\operatorname{dim} W^{c u}(p)$, if $\widehat{p}$ is a hyperbolic equilibrium point, see [7]. If $\widehat{p}$ is a subcritical Hopf equilibrium point, then $\operatorname{dim} W^{c u}(\widehat{p})<2$ or $\operatorname{dim} W^{c}(\widehat{p})<2$, which is a contradiction since the central manifold of a subcritical Hopf equilibrium point has at least dimension 2. Let $\widehat{p}$ be a hyperbolic equilibrium point and, therefore, $\operatorname{dim} W^{u}(\widehat{p})<2$. It follows that $\operatorname{dim} W^{u}(\widehat{p})=1$, since hyperbolic equilibrium points of type-zero can not belong to the boundary of the stability region. Therefore, by item 1(i) of Theorem 4.2, $W^{u}(\widehat{p}) \cap A\left(x^{s}\right) \neq \emptyset$. Let $y \in W^{u}(\widehat{p}) \cap A\left(x^{s}\right)$ and $B(y, \varepsilon)$ be an open ball of radius $\varepsilon>0$ centered at $y$. Since $A\left(x^{s}\right)$ is an open set, then $B(y, \varepsilon) \subset A\left(x^{s}\right)$ for $\varepsilon$ sufficiently small. Let $N^{c}$ be a neighborhood of $q$ at $W^{c}(p)$. The neighborhood $N^{c}$ contains a transversal section $D$ of $W^{s}(\widehat{p})$ at the point $q$ with $\operatorname{dimension} \operatorname{dim} D=2$. By $\lambda$-lemma for non hyperbolic equilibrium points, see [6], there is a point $w \in D$ and a time $t_{w}>0$ such that $\varphi\left(t_{w}, w\right) \in N^{c}$. As $A\left(x^{s}\right)$ is invariant, then $w \in A\left(x^{s}\right)$. Therefore, $w \in W^{c}(p) \cap A\left(x^{s}\right)$ and, consequently, $W^{c}(p) \cap A\left(x^{s}\right) \neq \emptyset$.

(2ii) $(\Longleftarrow)$ Suppose that $W^{s}(p) \subset \partial A\left(x^{s}\right)$. Since $p \in W^{s}(p)$, then $p \in \partial A\left(x^{s}\right)$.

$(\Longrightarrow)$ Suppose now that $p \in \partial A\left(x^{s}\right)$. By item 2(i) of Theorem 4.2, we can conclude that $W^{c}(p) \cap A\left(x^{s}\right) \neq \emptyset$. Let $y \in W^{c}(p) \cap A\left(x^{s}\right)$. Since $y \in W^{c}(p)$, then there is $T<0$ such that $\varphi(T, y) \in W_{l o c}^{c}(p)$. Let $z=\varphi(T, y)$. Since $y \in A\left(x^{s}\right)$ and $A\left(x^{s}\right)$ is invariant, then $z \in A\left(x^{s}\right)$. It follows that $z \in W_{l o c}^{c}(p) \cap A\left(x^{s}\right)$. Let $B(z, \varepsilon)$ be an open ball of radius $\varepsilon>0$ centered at $z$ where $\varepsilon$ is an arbitrarily small number. Let $\widehat{q}$ be an arbitrary point of $W^{s}(p)$. In particular, for some $\bar{T}>0$ we have $\widetilde{q}=\varphi(\bar{T}, \widehat{q}) \in W_{l o c}^{s}(p)$. Let $S$ be a disk at point $\widetilde{q}$ of $\operatorname{dim} S=2$ transverse to $W_{l o c}^{s}(p)$. By $\lambda$-lemma for non hyperbolic equilibrium points, see [6], there is a point $w \in S$ and a time $t_{w}>0$ such that $\varphi\left(t_{w}, w\right) \in B(z, \varepsilon)$. Since $A\left(x^{s}\right)$ is invariant, then $w \in A\left(x^{s}\right)$. Since $\varepsilon$ and the disk $S$ can be chosen arbitrarily small, then there are points at $A\left(x^{s}\right)$ arbitrarily close to $\widetilde{q}$. Consequently, $\widetilde{q} \in \overline{A\left(x^{s}\right)}$. Since $W_{l o c}^{s}(p) \cap A\left(x^{s}\right)=\emptyset$, then $\widetilde{q} \in \partial A\left(x^{s}\right)$. Since $\partial A\left(x^{s}\right)$ is invariant, $\widehat{q}=\varphi(-\bar{T}, \widetilde{q}) \in \partial A\left(x^{s}\right)$. As the choice of $\widehat{q}$ at $W^{s}(p)$ was arbitrary, then we can conclude that $W^{s}(p) \subset \partial A\left(x^{s}\right)$. 
(3i) $(\Longleftarrow)$ Suppose that $W^{c u}(p) \cap A\left(x^{s}\right) \neq \varnothing$. Since $A\left(x^{s}\right) \subset \overline{A\left(x^{s}\right)}$, then $\left(W_{l o c}^{c u}(p) \backslash\{p\}\right) \cap$ $\overline{A\left(x^{s}\right)} \neq \emptyset$. Therefore, by item (ii) of Theorem 4.1, we have that $p \in \partial A\left(x^{s}\right)$.

$(\Longrightarrow)$ Suppose that $p \in \partial A\left(x^{s}\right)$. By Theorem 4.1, we can conclude that $\left(W_{l o c}^{c u}(p) \backslash\{p\}\right) \cap$ $\overline{A\left(x^{s}\right)} \neq \varnothing$. Consequently, $\left(W^{c u}(p) \backslash\{p\}\right) \cap \overline{A\left(x^{s}\right)} \neq \emptyset$, since $W_{l o c}^{c u}(p) \subset W^{c u}(p)$. Let us show, under assumptions (A1"), (A2") and (A3) that $\left(W^{c u}(p) \backslash\{p\}\right) \cap \overline{A\left(x^{s}\right)} \neq \varnothing$ implies $W^{c u}(p) \cap A\left(x^{s}\right) \neq \emptyset$. Let $q \in\left(W^{c u}(p) \backslash\{p\}\right) \cap \overline{A\left(x^{s}\right)}$. If $q \in A\left(x^{s}\right)$, then there is nothing to be proved. Suppose that $q \in \partial A\left(x^{s}\right)$. From condition (A3), there is an equilibrium point $\widehat{p} \in \partial A\left(x^{s}\right)$ such that $\varphi(t, q) \rightarrow \widehat{p}$ as $t \rightarrow+\infty$. By the dimension of the unstable manifold of the equilibrium point, see [7], we can conclude that $\widehat{p} \neq p$. Then $\widehat{p}$ is a hyperbolic equilibrium point or subcritical Hopf equilibrium point and we conclude that $\operatorname{dim} W^{c u}(\widehat{p})<\operatorname{dim} W^{c u}(p)$ or $\operatorname{dim} W^{c}(\widehat{p})<\operatorname{dim} W^{c u}(p)$, if $\widehat{p}$ is a subcritical Hopf equilibrium point or $\operatorname{dim} W^{u}(\widehat{p})<$ $\operatorname{dim} W^{c u}(p)$, if $\widehat{p}$ is a hyperbolic equilibrium point, see [7]. If $\widehat{p}$ is a subcritical Hopf equilibrium point, then $\operatorname{dim} W^{c u}(\widehat{p})<3$ or $\operatorname{dim} W^{c}(\widehat{p})<3$. It follows that $\operatorname{dim} W^{c}(\widehat{p})=2$, since the central manifold of a subcritical Hopf equilibrium point has at least dimension 2. Therefore, by item 2(i) of Theorem 4.2, $W^{c}(\widehat{p}) \cap A\left(x^{s}\right) \neq \emptyset$. Let $y \in W^{c}(\widehat{p}) \cap A\left(x^{s}\right)$ and $B(y, \varepsilon)$ be an open ball of radius $\varepsilon>0$ centered at $y$. Since $A\left(x^{s}\right)$ is an open set, then $B(y, \varepsilon) \subset A\left(x^{s}\right)$ for $\varepsilon$ suficiently small. Let $N^{c}$ be a neighborhood of $q$ at $W^{c u}(p)$. The neighborhood $N^{c}$ contains a transversal section $D$ of $W^{s}(\widehat{p})$ at the point $q$ with dimension $\operatorname{dim} D=2$. By $\lambda$-lemma for non hyperbolic equilibrium points, see [6], there is a point $w \in D$ and a time $t_{w}>0$ such that $\varphi\left(t_{w}, w\right) \in N^{c}$. Since $A\left(x^{s}\right)$ is invariant set, then $w \in A\left(x^{s}\right)$. Therefore, $w \in W^{c u}(p) \cap A\left(x^{s}\right)$ and, consequently, $W^{c u}(p) \cap A\left(x^{s}\right) \neq \emptyset$.

If $\widehat{p}$ is a hyperbolic equilibrium point and, therefore, $\operatorname{dim} W^{u}(\widehat{p})<3$, it follows that $\operatorname{dim}$ $W^{u}(\widehat{p})=1$ or $\operatorname{dim} W^{u}(\widehat{p})=2$, since hyperbolic equilibrium points of type-zero can not belong to the boundary of the stability region. Thus, by item 1(i) of Theorem 4.2, $W^{u}(\widehat{p}) \cap A\left(x^{s}\right) \neq \emptyset$. Let $y \in W^{u}(\widehat{p}) \cap A\left(x^{s}\right)$ and $B(y, \varepsilon)$ be an open ball of radius $\varepsilon>0$ centered at $y$. Since $A\left(x^{s}\right)$ is an open set, then $B(y, \varepsilon) \subset A\left(x^{s}\right)$ for $\varepsilon$ suficiently small. Let $N^{c}$ be a neighborhood of $q$ at $W^{c u}(p)$. The neighborhood $N^{c}$ contains a transversal section $D$ of $W^{s}(\widehat{p})$ at the point $q$ with dimension $\operatorname{dim} D=2$. By $\lambda$-lemma, see [6], there is a point $w \in D$ and a time $t_{w}>0$ such that $\varphi\left(t_{w}, w\right) \in N^{c}$. Since $A\left(x^{s}\right)$ is invariant set, then $w \in A\left(x^{s}\right)$. Therefore, $w \in W^{c u}(p) \cap A\left(x^{s}\right)$ and, consequently, $W^{c u}(p) \cap A\left(x^{s}\right) \neq \emptyset$.

(3ii) $(\Longleftarrow)$ Suppose that $W^{s}(p) \subset \partial A\left(x^{s}\right)$. Since $p \in W^{s}(p)$, then $p \in \partial A\left(x^{s}\right)$.

$(\Longrightarrow)$ Suppose that $p \in \partial A\left(x^{s}\right)$. By item 3(i) of Theorem 4.2, we can conclude that $W^{c u}(p) \cap$ $A\left(x^{s}\right) \neq \varnothing$. Let $y \in W^{c u}(p) \cap A\left(x^{s}\right)$. Since $y \in W^{c u}(p)$, then there is $T<0$ such that $\varphi(T, y) \in W_{l o c}^{c u}(p)$. Let $z=\varphi(T, y)$. Since $y \in A\left(x^{s}\right)$ and $A\left(x^{s}\right)$ is invariant, then $z \in A\left(x^{s}\right)$. It follows that $z \in W_{l o c}^{c u}(p) \cap A\left(x^{s}\right)$. Let $B(z, \varepsilon)$ be an open ball of radius $\varepsilon>0$ centered at $z$ where $\varepsilon$ is an arbitrarily small number. Let $\widehat{q}$ be an arbitrary point of $W^{s}(p)$. In particular, for some $\bar{T}>0$ we have $\widetilde{q}=\varphi(\bar{T}, \widehat{q}) \in W_{l o c}^{s}(p)$. Let $S$ be a disk at point $\widetilde{q}$ of $\operatorname{dim} S=3$ transverse to $W_{l o c}^{s}(p)$. By $\lambda$-lemma for non hyperbolic equilibrium points, see [6], there is a point $w \in S$ and a time $t_{w}>0$ such that $\varphi\left(t_{w}, w\right) \in B(z, \varepsilon)$. Since $A\left(x^{s}\right)$ is an invariant, then $w \in A\left(x^{s}\right)$. 
Since $\varepsilon$ and the disk $S$ can be chosen arbitrarily small, then there are points at $A\left(x^{s}\right)$ arbitrarily close to $\widetilde{q}$. Consequently, $\widetilde{q} \in \overline{A\left(x^{s}\right)}$. Since $W_{l o c}^{s}(p) \cap A\left(x^{s}\right)=\emptyset$, then $\widetilde{q} \in \partial A\left(x^{s}\right)$. Since $\partial A\left(x^{s}\right)$ is invariant, $\widehat{q}=\varphi(-\bar{T}, \widetilde{q}) \in \partial A\left(x^{s}\right)$. As the choice of $\widehat{q}$ at $W^{s}(p)$ was arbitrary, then we can conclude that $W^{s}(p) \subset \partial A\left(x^{s}\right)$.

The next theorem shows the characterization of hyperbolic and subcritical Hopf equilibrium points on the boundary of stability region for type- $k$, with $1 \leq k \leq n-2$.

Theorem 4.3. (Type-k equilibrium points on $\left.\partial A\left(x^{s}\right)\right)$ : Let $A\left(x^{s}\right)$ be the stability region of an asymptotically stable equilibrium point $x^{s}$ of (2.1) and suppose the assumptions (A1"), (A2") and $(\mathbf{A 3})$ are held. Let $p$ be a type-k subcritical Hopf equilibrium point, with $1 \leq k \leq n-2$, and $x^{\star}$ be a type- $k^{\prime}$ hyperbolic equilibrium point, with $k^{\prime} \leq n$, of (2.1). Then

$$
\text { (i) } \begin{aligned}
& p \in \partial A\left(x^{s}\right) \Longleftrightarrow W^{c u}(p) \cap A\left(x^{s}\right) \neq \emptyset \\
& x^{\star} \in \partial A\left(x^{s}\right) \Longleftrightarrow W^{u}\left(x^{\star}\right) \cap A\left(x^{s}\right) \neq \emptyset \\
& \text { (ii) } \quad p \in \partial A\left(x^{s}\right) \Longleftrightarrow W^{s}(p) \subset \partial A\left(x^{s}\right) \\
& x^{\star} \in \partial A\left(x^{s}\right) \Longleftrightarrow W^{s}\left(x^{\star}\right) \subset \partial A\left(x^{s}\right)
\end{aligned}
$$

Proof. (i) $(\Longleftarrow)$ The proof is analogous to the proof of the previous theorem and will be omitted.

$(\Longrightarrow)$ We will demonstrate the theorem by using finite induction on the dimension of $W^{c u}(x)$ or $W^{u}(x)$ if $x \in \partial A\left(x^{s}\right)$ is a subcritical Hopf equilibrium point or hyperbolic equilibrium point. If $\operatorname{dim} W^{u}(x)=1$, then by Theorem 4.2 we know that $W^{u}(x) \cap A\left(x^{s}\right) \neq \emptyset$ or $W^{c u}(x) \cap A\left(x^{s}\right) \neq \emptyset$. Suppose that $W^{u}(x) \cap A\left(x^{s}\right) \neq \emptyset$ or $W^{c u}(x) \cap A\left(x^{s}\right) \neq \emptyset$ for all equilibrium points $x$ at the boundary $\partial A\left(x^{s}\right)$ with $\operatorname{dim} W^{u}(x) \leq k$. Now, we suppose that $\operatorname{dim} W^{u}(x)=k+1$. By Theorem 4.1 or Theorem 3.7 of [3], we can conclude that $\left(W_{l o c}^{i}(x) \backslash\{x\}\right) \cap \overline{A\left(x^{s}\right)} \neq \emptyset$, for $i=c u$ or $i=u$ respectively. Consequently $\left(W^{i}(x) \backslash\{x\}\right) \cap \overline{A\left(x^{s}\right)} \neq \emptyset$, since $W_{l o c}^{i}(x) \subset W^{i}(x)$. Let us show, under assumptions (A1"), (A2") and (A3) that $\left(W^{i}(x) \backslash\{x\}\right) \cap \overline{A\left(x^{s}\right)} \neq \emptyset$ implies $W^{i}(x) \cap$ $A\left(x^{s}\right) \neq \emptyset$. Let $q \in\left(W^{i}(x) \backslash\{p\}\right) \cap \overline{A\left(x^{s}\right)}$. If $q \in A\left(x^{s}\right)$, there is nothing to be proved. Suppose that $q \in \partial A\left(x^{s}\right)$. From condition (A3), there is an equilibrium point $\widehat{p} \in \partial A\left(x^{s}\right)$ such that $\varphi(t, q) \rightarrow \widehat{p}$ as $t \rightarrow+\infty$. From (A1"), it is concluded that $\widehat{p}$ is a hyperbolic equilibrium point or subcritical Hopf equilibrium point. By the dimension of the unstable manifold of the equilibrium point, see [7], if $x$ is a type- $k$ hyperbolic equilibrium point or subcritical Hopf equilibrium point, with $1 \leq k \leq n-3$, or a type- $(n-2)$ subcritical Hopf equilibrium point respectively, we conclude that $\operatorname{dim} W^{j}(\widehat{p})<\operatorname{dim} W^{i}(x)$, for $j=u$ if $\widehat{p}$ is a hyperbolic equilibrium point or $j=c u$ if $\widehat{p}$ is a subcritical equilibrium point. Let $\widehat{p}$ be a hyperbolic equilibrium point. Since $\operatorname{dim} W^{u}(\widehat{p})<\operatorname{dim} W^{i}(x)$, then we conclude that $\operatorname{dim} W^{u}(\widehat{p}) \leq k$. Thus, by induction hypothesis $W^{u}(\widehat{p}) \cap A\left(x^{s}\right) \neq \emptyset$. Let $y \in W^{u}(\widehat{p}) \cap A\left(x^{s}\right)$ and $B(y, \varepsilon)$ be an open ball of radius $\varepsilon>0$ centered at $y$. Since $A\left(x^{s}\right)$ is an open set, then $B(y, \varepsilon) \subset A\left(x^{s}\right)$ for $\varepsilon$ suficiently small. Let $N^{i}$ be a neighborhood of $q$ at $W^{i}(x)$. The neighborhood $N^{i}$ contains a transversal section $D$ of $W^{s}(\widehat{p})$ at the point $q$ with $\operatorname{dimension} \operatorname{dim} D \leq k$. By $\lambda$-lemma, see [6], there is a point $w \in D$ and a 
time $t_{w}>0$ such that $\varphi\left(t_{w}, w\right) \in N^{i}$. Since $A\left(x^{s}\right)$ is a invariant, then $w \in A\left(x^{s}\right)$. Therefore, $w \in W^{i}(x) \cap A\left(x^{s}\right)$ and, consequently, $W^{i}(x) \cap A\left(x^{s}\right) \neq \emptyset$.

If $\widehat{p}$ is a subcritical Hopf equilibrium point and since $\operatorname{dim} W^{c u}(\widehat{p})<\operatorname{dim} W^{i}(x)$, then we also conclude that $\operatorname{dim} W^{c u}(\widehat{p}) \leq k$. Thus, by induction hypothesis $W^{c u}(\widehat{p}) \cap A\left(x^{s}\right) \neq \emptyset$. Let $y \in$ $W^{c u}(\widehat{p}) \cap A\left(x^{s}\right)$ and $B(y, \varepsilon)$ be an open ball of radius $\varepsilon>0$ centered at $y$. Since $A\left(x^{s}\right)$ is an open set, then $B(y, \varepsilon) \subset A\left(x^{s}\right)$ for $\varepsilon$ suficiently small. Let $N^{i}$ be a neighborhood of $q$ at $W^{i}(x)$. The neighborhood $N^{i}$ contains a transversal section $D$ of $W^{s}(\widehat{p})$ at the point $q$ with dimension $\operatorname{dim} D \leq k$. By $\lambda$-lemma for non hyperbolic equilibrium points, see [6], there is a point $w \in D$ and a time $t_{w}>0$ such that $\varphi\left(t_{w}, w\right) \in N^{i}$. Since $A\left(x^{s}\right)$ is an invariant, then $w \in A\left(x^{s}\right)$. Thus, $w \in W^{i}(x) \cap A\left(x^{s}\right)$ and consequently, $W^{i}(x) \cap A\left(x^{s}\right) \neq \emptyset$.

(ii) The proof is analogous to the proof of Theorem 4.2 and will be omitted.

The next theorem provides a complete characterization of the boundary of the stability region when there are subcritical Hopf equilibrium points in $\partial A\left(x^{s}\right)$.

Theorem 4.4. (Stability Boundary Characterization): Let $x^{s}$ be an asymptotically stable equilibrium point of (2.1) and $A\left(x^{s}\right)$ be its stability region. If assumptions (A1") and (A3) are satisfied, then

$$
\partial A\left(x^{s}\right) \subset \bigcup_{i} W^{s}\left(x_{i}\right) \bigcup_{j} W^{s}\left(p_{j}\right)
$$

where $x_{i}$ are the hyperbolic equilibrium points and $p_{j}$ are the subcritical Hopf equilibrium points on $\partial A\left(x^{s}\right), i, j=1,2, \ldots$ If supposition (A2") is satisfied, then

$$
\partial A\left(x^{s}\right)=\bigcup_{i} W^{s}\left(x_{i}\right) \bigcup_{j} W^{s}\left(p_{j}\right) .
$$

Proof. Let $q \in \partial A\left(x^{s}\right)$. By assumption (A3), we can assert that there is an equilibrium point $x$ such that $\varphi(t, q) \rightarrow x$ when $t \rightarrow+\infty$. By assumption (A1"), we can assert that $x$ is either a hyperbolic equilibrium point $x_{i}$ or a subcritical Hopf equilibrium point $p_{j}$, namely $x=x_{i}$ or $x=p_{j}$ for some $i, j$. Therefore, we conclude that $q \in \bigcup_{i} W^{s}\left(x_{i}\right) \bigcup_{j} W^{s}\left(p_{j}\right)$. Therefore, $\partial A\left(x^{s}\right) \subset \bigcup_{i} W^{s}\left(x_{i}\right) \bigcup_{j} W^{s}\left(p_{j}\right)$. By Theorems 4.2 and 4.3, we know that $W^{s}\left(x_{i}\right) \subset \partial A\left(x^{s}\right)$ and $W^{s}\left(p_{j}\right) \subset \partial A\left(x^{s}\right)$. Thus, $\bigcup_{i} W^{s}\left(x_{i}\right) \bigcup_{j} W^{s}\left(p_{j}\right) \subset \partial A\left(x^{s}\right)$ and, therefore,

$$
\partial A\left(x^{s}\right)=\bigcup_{i} W^{s}\left(x_{i}\right) \bigcup_{j} W^{s}\left(p_{j}\right) .
$$

\section{CONCLUSION}

In this paper, we studied the characterization of the boundary of stability regions of nonlinear dynamical autonomous systems in the presence of subcritical Hopf equilibrium points. Necessary and sufficient conditions were offered for a hyperbolic equilibrium point and a Hopf subcritical equilibrium point belonging to the boundary of the stability region. The characterization of the 
boundary of the stability region proposed in this paper is a generalization of the characterizations in the literature allowing the presence of a particular type of non-hyperbolic equilibrium point on the boundary of the stability region. Exploring the characterizations developed in this work, we hope in the near future, to understand how the stability region behaves when local bifurcations of type Hopf occur on the boundary of stability region.

\section{ACKNOWLEDGEMENT}

The authors gratefully acknowledge the support and funds provided by CNPQ under the grant 305486/2013-6.

RESUMO. Uma caracterização completa da fronteira da região de estabilidade de uma classe de sistemas dinâmicos autônomos não lineares é desenvolvida admitindo a existência de pontos de equilíbrio não-hiperbólicos do tipo Hopf Subcríticos na fronteira da região de estabilidade. A caracterização da região de estabilidade neste trabalho é uma extensão da caracterização já desenvolvida na literatura, que consideram somente ponto de equilíbrio hiperbólico. Sob a condição de transversalidade, mostra-se que a fronteira da região de estabilidade é composta pelas variedades estáveis de todos os pontos de equilíbrio na fronteira da região de estabilidade, incluindo as variedades estáveis dos pontos de equilíbrio Hopf Subcríticos do tipo $k$, com $0 \leq k \leq n-2$, que pertencem à fronteira da região de estabilidade.

Palavras-chave: sistemas dinâmicos, sistemas não lineares, região de estabilidade, fronteira da região de estabilidade, ponto de equilíbrio Hopf subcrítico.

\section{REFERENCES}

[1] F.M. Amaral \& L.F.C. Alberto. Stability boundary characterization of nonlinear autonomous dynamical systems in the presence of a type-zero saddle-node equilibrium point. TEMA - Tendências em Matemática Aplicada e Computacional, [S.1], 11 (2010), 111-120.

[2] F.M. Amaral \& L.F.C. Alberto. Stability region bifurcations of nonlinear autonomous dynamical systems: Type-zero saddle-node bifurcations. International Journal of Robust and Nonlinear Control, 21(6) (2011), 591-612.

[3] H.D. Chiang, M.W. Hirsch \& F.F. Wu. Stability region of nonlinear autonomous dynamical systems. IEEE Transactions on Automatic Control, 33(1) (1988), 16-27.

[4] H.D. Chiang, F.F. Wu \& P.P. Varaiya. Foundations of direct methods for power system transient stability analysis. IEEE Transactions on Circuits and Systems-I, 34(2) (1987), 160-173.

[5] H.D. Chiang \& L.F.C. Alberto. Stability Regions of Nonlinear Dynamical Systems: Theory, Estimation, and Applications, Cambridge University Press (2015).

[6] J. Palis. On Morse-Smale dynamical systems. Topology, 8(4) (1969), 385-405. 
[7] J. Palis Jr \& Welington Melo. Geometric Theory of Dynamical Systems: An Introduction, Springer Science \& Business Media (2012).

[8] J.R.R. Gouveia Jr, L.F.C. Alberto \& F.M. Amaral. Supercritical Hopf equilibrium points on the Boundary of the Stability Region, in "Decision and Control (CDC)", 2013 IEEE 52nd Annual Conference on. IEEE, (2013), 5252-5257.

[9] J.R.R. Gouveia Jr, F.M. Amaral \& L.F.C. Alberto. Stability boundary characterization of nonlinear autonomous dynamical systems in the presence of a supercritical Hopf equilibrium point. International Journal of Bifurcation and Chaos, 23(12) (2013), 1350196-1-1350196-13.

[10] J. Sotomayor. Generic bifurcations of dynamical systems, Dynamical Systems, New York: Academic Press (1973).

[11] J. Sotomayor \& M.A. Teixeira. Vector Fields near the boundary of a 3-manifold. Lect. Notes in Math., Springer Verlag, 1331 (1988), 169-195.

[12] L. Perko. Differential equations and dynamical systems, Springer (1991).

[13] M.W. Hirsch, C.C. Pugh \& M. Shub. Invariant manifolds. Bull. Amer. Math. Soc., 76(5) (1970), $1015-1019$.

[14] N.A. Tsolas, A. Arapostathis \& P.P. Varaya. A Structure presereving energy function for power system transient stability analysis. IEEE Transactions on Circuits and Systems, 32 (1985), 1041-1049.

[15] S. Smale. Differentiable dynamical systems. Bulletin of the American Mathematical Society, 73(6) (1967), 747-817.

[16] S. Wiggins. Introduction to Applied Nonlinear Dynamical Systems and Chaos, Springer Verlag, New York (1989).

[17] V. Guillemin \& A. Pollack. Differential Topology, Englewood Cliffs, NJ: Prentice-Hall (1974).

[18] Y.A. Kuznetsov. Elements of Applied Bifurcation Theory, Vol. 112. Springer (2003). 